\title{
On a Unique Ergodicity of Some Markov Processes
}

\author{
Rafał Kapica · Tomasz Szarek • Maciej Ślęczka
}

Received: 26 February 2011 / Accepted: 21 June 2011 / Published online: 12 July 2011

(C) The Author(s) 2011. This article is published with open access at Springerlink.com

\begin{abstract}
It is proved that the sufficient condition for the uniqueness of an invariant measure for Markov processes with the strong asymptotic Feller property formulated by Hairer and Mattingly (Ann Math 164(3):993-1032, 2006) entails the existence of at most one invariant measure for e-processes as well. Some application to timehomogeneous Markov processes associated with a nonlinear heat equation driven by an impulsive noise is also given.
\end{abstract}

Keywords Ergodicity of Markov families • Invariant measures •

Stochastic heat equations

Mathematics Subject Classifications (2010) Primary 60J25 • 60H15;

Secondary $76 \mathrm{~N} 10$

\section{Introduction}

In the 60's Khas'minskii showed the uniqueness of an invariant measure for Markov processes under the strong Feller condition (see [4]). Some ideas of its consideration were taken from [2]. This result appeared to be a very useful tool in the theory

This work has been partly supported by Polish Ministry of Science and Higher Education Grants N N 201419139 (TS).

R. Kapica · M. Ślȩczka $(\varangle)$

Institute of Mathematics, University of Silesia, Bankowa 14, 40-007 Katowice, Poland

e-mail: sleczka@ux2.math.us.edu.pl

R. Kapica

e-mail: rkapica@ux2.math.us.edu.pl

T. Szarek

Institute of Mathematics, University of Gdańsk, Wita Stwosza 57, 80-952 Gdańsk, Poland e-mail: szarek@intertele.pl 
of Markov processes corresponding to stochastic differential equations driven by some noise, mostly a Wiener one. Indeed, if the noise is sufficiently regular, then we may prove that the process satisfies the strong Feller property (see [7-9]). Recently Priola and Zabczyk have established the strong Feller property for Markov processes corresponding to SPDEs driven by cylindrical stable processes (see [12]). To be precise, the strong Feller property means that the process given by a semigroup of operators $\left(P_{t}\right)_{t \geq 0}$ acting on some Polish space $X$ is such that $P_{t_{0}} \phi$ is continuous for every bounded Borel-measurable function $\phi: X \rightarrow \mathbb{R}$ and some $t_{0}>0$. To obtain the strong Feller property on some Hilbert space $\mathcal{H}$ it is usually assumed that the process under consideration fulfills the following condition: for any $\phi: \mathcal{H} \rightarrow \mathbb{R}$ bounded and with the bounded Fréchet derivative $\nabla \phi$ we have

$$
\left|\nabla P_{t} \phi(x)\right| \leq C(\|x\|) \sup _{y \in \mathcal{H}}|\phi(y)|,
$$

where $C: \mathbb{R}_{+} \rightarrow \mathbb{R}$ is a fixed nondecreasing function (see [1]). Hairer and Mattingly introduced the concept of asymptotic strong Feller operators. We do not recall it here and refer the reader to [3]. It is worth mentioning here that the asymptotically strong Feller property is implied by a condition being, at first sight, a slight modification of condition (1.1). The condition has the form (see [3, Proposition 3.12]):

$$
\left|\nabla P_{t_{n}} \phi(x)\right| \leq C(\|x\|)\left(\sup _{y \in \mathcal{H}}|\phi(y)|+\delta_{n} \sup _{y \in \mathcal{H}}\|\nabla \phi(y)\|\right),
$$

where $C, \phi$ are as above but $\left(t_{n}\right)_{n \geq 1}$ and $\left(\delta_{n}\right)_{n \geq 1}$ are two positive sequences with $\left(t_{n}\right)_{n \geq 1}$ nondecreasing and $\left(\delta_{n}\right)_{n \geq 1}$ converging to zero. Condition (1.2) is more than a cosmetic change, in fact it allowed Hairer and Mattingly to prove uniqueness of an invariant measure for a broad class of stochastic differential equations including the Navier-Stokes equation driven by degenerate noise (see [3]). To do this they show that every Markov process which is (weakly) irreducible and asymptotically strong Feller possesses at most one invariant measure.

In almost the same time Lasota and the second author set forth the concept of an e-process formulating for it a criterion for the existence of an invariant measure (see [6]). Sufficient conditions for its uniqueness were given in [5]. Recall that a stochastic process on $X$ given by a semigroup $\left(P_{t}\right)_{t \geq 0}$ is called an $e$-process if it satisfies the eproperty: for any point $x \in X$ and an arbitrary Lipschitz bounded function $\phi: X \rightarrow$ $\mathbb{R}$ the family $\left\{P_{t} \phi\right\}_{t \geq 0}$ is equicontinuous in $x$, i.e.

$$
\lim _{y \rightarrow x} \sup _{t \geq 0}\left|P_{t} \phi(x)-P_{t} \phi(y)\right|=0 .
$$

It was shown (see [5]) that to verify the e-property in the case of some Hilbert space $\mathcal{H}$ it is enough to prove that there exists $C: \mathbb{R}_{+} \rightarrow \mathbb{R}_{+}$such that

$$
\left|\nabla P_{t} \phi(x)\right| \leq C(\|x\|)\left(\sup _{y \in \mathcal{H}}|\phi(y)|+\sup _{y \in \mathcal{H}}\|\nabla \phi(y)\|\right)
$$

for any $\phi \in C^{1}(\mathcal{H})$ and $t>0$.

Since there exist a large class of e-processes with no asymptotically strong Feller property (see Remark 6 in [5]) it seemed plausible to prove an analogous criterion for the uniqueness of an invariant measure as that contained in [3]. This will be done in the first part of our paper. Actually, we shall prove that any weakly irreducible 
e-process admits at most one invariant measure (see Theorem 2). To show it we have introduced the concept of a point which is to generate a given measure. Generally speaking, the averages of iterates of this measure behaves approximately as those starting with the Dirac measure supported in the given point.

In the second part of the paper we reformulate our main result in the setting of SPDE's providing a simple criterion on the uniqueness of an invariant measure. Further, we are concerned with the process corresponding to the heat equation driven by an impulsive noise in the borderline case when the equation becomes merely weakly dissipative and not dissipative. The studied equation is a slight modification of the equation investigated by Peszat and Zabczyk in [10] (see also [6]). The difference lies in assuming that the impulsive white noise affects the system in every direction independently. Having this we may show that the process corresponding to the stochastic heat equation is an e-process. Moreover, the process under consideration is also weakly irreducible. From the proven theorem it follows then that it has at most one invariant measure (Proposition 3).

\section{General Theorem}

Let $X$ be a Polish space and let $\mathcal{B}(X)$ denote the $\sigma$-algebra of all Borel subsets of $X$. By $B_{b}(X)$ we denote the space of bounded Borel-measurable functions equipped with the supremum norm. Let $\left(P_{t}\right)_{t \geq 0}$ be the Markovian semigroup defined on $B_{b}(X)$. For each $t \geq 0$ we have $P_{t} \mathbf{1}=\mathbf{1}$ and $P_{t} \psi \geq 0$ if $\psi \geq 0$. Throughout this paper we shall assume that the semigroup is Feller, i.e. $P_{t}\left(C_{b}(X)\right) \subset C_{b}(X)$ for all $t>0$ and stochastically continuous, i.e. for $\psi \in C_{b}(X)$ and $x \in X$ we have $\lim _{t \rightarrow 0+} P_{t} \psi(x)=\psi(x)$. Here $C_{b}(X)$ is the subspace of bounded continuous functions. By $\mathcal{L}_{b}(X)$ we will denote the subspace of all bounded Lipschitz functions.

By $\pi^{t}: X \times \mathcal{B}(X) \rightarrow[0,1], t \geq 0$, we denote the transition probability for $\left(P_{t}\right)_{t \geq 0}$, i.e. $\pi^{t}(x, A)=P_{t} \mathbf{1}_{A}(x)$.

Let $\mathcal{M}$ denote the space of all finite Borel measures on $X$, and let $\mathcal{M}_{1}$ stand for the subspace of $\mathcal{M}$ of all probability measures. By supp $\mu$ we denote the support of the measure $\mu$. Recall that the total variation norm of a finite signed measure $\mu \in$ $\mathcal{M}-\mathcal{M}$ is given by $\|\mu\|_{T V}=\mu^{+}(X)+\mu^{-}(X)$, where $\mu=\mu^{+}-\mu^{-}$is the Jordan decomposition of $\mu$.

We say that $\mu_{*} \in \mathcal{M}_{1}$ is invariant for $\left(P_{t}\right)_{t \geq 0}$ if

$$
\int_{X} P_{t} \psi(x) \mu_{*}(\mathrm{~d} x)=\int_{X} \psi(x) \mu_{*}(\mathrm{~d} x)
$$

for every $\psi \in B_{b}(X)$ and $t \geq 0$. Alternatively, we can say that $P_{t}^{*} \mu_{*}=\mu_{*}$ for all $t \geq 0$, where $\left(P_{t}^{*}\right)_{t \geq 0}$ denotes the semigroup dual to $\left(P_{t}\right)_{t \geq 0}$, i.e. for a given Borel measure $\mu$ and $t \geq 0$ we set

$$
P_{t}^{*} \mu(A):=\int_{X} P_{t} \mathbf{1}_{A}(x) \mu(\mathrm{d} x) \quad \text { for } A \in \mathcal{B}(X) .
$$

If $\mu \in \mathcal{M}_{1}$ is invariant we may associate with the semigroup $\left(P_{t}\right)_{t \geq 0}$ the dynamical system $\left(\Omega, \mathcal{F},\left(\Theta_{t}\right)_{t \in \mathbb{R}}, \mathbb{P}_{\mu}\right)$ given in the following way:

- $\Omega=X^{\mathbb{R}}$,

- $\mathcal{F}=\mathcal{B}(X)^{\mathbb{R}}$, 
- $\quad\left(\Theta_{t}\right)_{t \in \mathbb{R}}$ is the group of invertible, measurable transformations from $\Omega$ to $\Omega$ given by the formula:

$$
\left(\Theta_{t} \omega\right)(s)=\omega(t+s) \quad \text { for } t, s \in \mathbb{R},
$$

- $\mathbb{P}_{\mu}$ is the unique measure, by the Kolmogorov extension theorem, such that

$$
\left.\mathbb{P}_{\mu}\left(\left\{\omega \in \Omega:\left(\omega\left(t_{1}\right), \ldots, \omega\left(t_{n}\right)\right) \in \Gamma\right)\right\}\right)=\mathbb{P}_{\mu}^{\left\{t_{1}, \ldots, t_{n}\right\}}(\Gamma),
$$

where

$$
\begin{aligned}
& P_{\mu}^{\left\{t_{1}, \ldots, t_{n}\right\}}(\Gamma) \\
& =\int_{X} \mu(\mathrm{d} x) \int_{X} \pi^{t_{1}}\left(x, \mathrm{~d} x_{1}\right) \int_{X} \pi^{t_{2}-t_{1}}\left(x_{1}, \mathrm{~d} x_{2}\right) \cdots \int_{X} \pi^{t_{n-1}-t_{n-2}}\left(x_{n-2}, \mathrm{~d} x_{n-1}\right) \\
& \quad \times \int_{X} \pi^{t_{n}-t_{n-1}}\left(x_{n-1}, \mathrm{~d} x_{n}\right) \mathbf{1}_{\Gamma}\left(x_{1}, \ldots, x_{n}\right) \\
& \quad \text { for } \Gamma \in \mathcal{B}(X)^{\left\{t_{1}, \ldots, t_{n}\right\}} \text { and } t_{1}<t_{2}<\ldots<t_{n} .
\end{aligned}
$$

An invariant measure $\mu \in \mathcal{M}_{1}$ is called ergodic if the system $\left(\Omega, \mathcal{F},\left(\Theta_{t}\right)_{t \in \mathbb{R}}, \mathbb{P}_{\mu}\right)$ is ergodic. We say that a measurable set $A \subset X$ is $\mu$-invariant if $\pi^{t}(x, A)=1$ for $\mu$-almost every $x \in A$ and $t \geq 0$. The following characterization of an ergodic measure is well known: $\mu$ is ergodic if and only if every $\mu$-invariant set $A$ is of $\mu$-measure 0 or 1 (see [1]).

For a given $t>0$ and $\mu \in \mathcal{M}_{1}$ define $Q^{t} \mu:=t^{-1} \int_{0}^{t} P_{s}^{*} \mu \mathrm{d} s$. When $t=0$ we adopt the convention $Q^{0} \mu:=\mu$. We also write $Q^{t}(x, \cdot)$ in the particular case when $\mu=\delta_{x}$. By $Q^{s, t} \mu$ for $s, t \geq 0$ we shall denote the composition $Q^{s}\left(Q^{t} \mu\right)$.

The crucial role in examining e-processes is played by the following set:

$$
\mathcal{T}:=\left\{x \in X: \text { the family of measures }\left(Q^{t}(x, \cdot)\right)_{t \geq 0} \text { is tight }\right\} .
$$

Recall that the existence of an invariant measure is equivalent to the condition $\mathcal{T} \neq \emptyset$. Moreover, if $\mu_{*}$ is invariant for $\left(P_{t}\right)_{t \geq 0}$, then supp $\mu_{*} \subset \mathcal{T}$ (see $\left.[5,13]\right)$.

Lemma 1 Assume that $\left(P_{t}\right)_{t \geq 0}$ has the e-property. Then for any $x \in \mathcal{T}$ the sequence $\left(Q^{t}(x, \cdot)\right)_{t \geq 0}$ weakly converges to some invariant measure.

Proof Fix $x \in \mathcal{T}$ and assume, contrary to our lemma, that the sequence $\left(Q^{t}(x, \cdot)\right)_{t \geq 0}$ does not converge. Since $\left(Q^{t}(x, \cdot)\right)_{t \geq 0}$ is tight, by the Prokhorov theorem we may find at least two different probability measures, say, $\mu_{*}, \mu^{*}$ and two sequences of positive reals $\left(s_{n}\right)_{n \geq 1}$ and $\left(t_{n}\right)_{n \geq 1}, \lim _{n \rightarrow \infty} s_{n}=\lim _{n \rightarrow \infty} t_{n}=+\infty$, such that $\left(Q^{s_{n}}(x, \cdot)\right)_{n \geq 1}$, $\left(Q^{t_{n}}(x, \cdot)\right)_{n \geq 1}$ weakly converge to $\mu_{*}$ and $\mu^{*}$, respectively.

Choose $f \in \mathcal{L}_{b}(X)$ such that $\int_{X} f \mathrm{~d} \mu_{*} \neq \int_{X} f \mathrm{~d} \mu^{*}$ and let $\varepsilon>0$ be such that $\left|\int_{X} f \mathrm{~d} \mu_{*}-\int_{X} f \mathrm{~d} \mu^{*}\right|>\varepsilon$.

Let $K \subset X$ be a compact set such that $Q^{t}(x, K)>1-\varepsilon /(6\|f\|)$ for all $t \geq 0$. Hence both the measure $\mu_{*}$ and $\mu^{*}$ on the set $K$ are grater than $1-\varepsilon /(6\|f\|)$, by the Alexandrov theorem. Passing to a subsequence if necessary, we may assume that on the set $K$ the sequence $\left(s_{n}^{-1} \int_{0}^{s_{n}} P_{s} f \mathrm{~d} s\right)_{n \geq 1}$ converges uniformly to some $\tilde{f}_{*} \in C(K)$, 
by the e-property and the Arzela-Ascoli theorem. Let $f_{*} \in C(X)$ be an extension of $\tilde{f}_{*}$ such that $\left\|f_{*}\right\|=\left\|\tilde{f}_{*}\right\|_{K}$. Obviously, $\left\|f_{*}\right\| \leq\|f\|$. From the definition of $K$ we obtain that $\left|\int_{X \backslash K} f_{*} \mathrm{~d} \mu^{*}\right|<\varepsilon / 6$ and $\left|\int_{X \backslash K}\left(s_{n}^{-1} \int_{0}^{s_{n}} P_{s} f \mathrm{~d} s\right) \mathrm{d} \mu^{*}\right|<\varepsilon / 6$. On the other hand, from the definition of $f_{*}$ we have $\int_{K} f_{*} \mathrm{~d} \mu^{*}=\lim _{n \rightarrow \infty} \int_{K}\left(s_{n}^{-1} \int_{0}^{s_{n}} P_{s} f \mathrm{~d} s\right) \mathrm{d} \mu^{*}$. Consequently, we obtain

$$
\left|\int_{X} f_{*} \mathrm{~d} \mu^{*}-\int_{X}\left(s_{n}^{-1} \int_{0}^{s_{n}} P_{s} f \mathrm{~d} s\right) \mathrm{d} \mu^{*}\right|<\varepsilon / 3
$$

for $n$ sufficiently large. Since $\mu^{*}$ is invariant, the second integral in the above formula equals $\int_{X} f \mathrm{~d} \mu^{*}$, which finally gives

$$
\left|\int_{X} f_{*} \mathrm{~d} \mu^{*}-\int_{X} f \mathrm{~d} \mu^{*}\right|<\varepsilon / 3 .
$$

Let $N \in \mathbb{N}$ be such that

$$
\left|\int_{X} f_{*} \mathrm{~d} \mu^{*}-\int_{X} f_{*} \mathrm{~d}\left(Q^{t_{N}}(x, \cdot)\right)\right|<\varepsilon / 3 .
$$

In the same manner as in the proof of formula (2.2) we may show that

$$
\left|\int_{X} f_{*} \mathrm{~d}\left(Q^{t_{N}}(x, \cdot)\right)-\int_{X}\left(s_{n}^{-1} \int_{0}^{s_{n}} P_{s} f \mathrm{~d} s\right) \mathrm{d}\left(Q^{t_{N}}(x, \cdot)\right)\right|<\varepsilon / 3
$$

for $n$ sufficiently large and hence

$$
\left|\int_{X} f_{*} \mathrm{~d}\left(Q^{t_{N}}(x, \cdot)\right)-\int_{X} f \mathrm{~d}\left(Q^{s_{n}, t_{N}}(x, \cdot)\right)\right|<\varepsilon / 3
$$

for $n$ sufficiently large. Since $\lim _{n \rightarrow \infty}\left\|Q^{s_{n}, t_{N}}(x, \cdot)-Q^{s_{n}}(x, \cdot)\right\|_{T V}=0$, by Lemma 2 in [5], we have

$$
\left|\int_{X} f_{*} \mathrm{~d}\left(Q^{t_{N}}(x, \cdot)\right)-\int_{X} f \mathrm{~d}\left(Q^{s_{n}}(x, \cdot)\right)\right|<\varepsilon / 3
$$

for $n$ sufficiently large and consequently

$$
\left|\int_{X} f_{*} \mathrm{~d}\left(Q^{t_{N}}(x, \cdot)\right)-\int_{X} f \mathrm{~d} \mu_{*}\right|<\varepsilon / 3 .
$$

Combining Eqs. 2.3-2.5 we obtain $\left|\int_{X} f \mathrm{~d} \mu^{*}-\int_{X} f \mathrm{~d} \mu_{*}\right|<\varepsilon$, contrary to the definition of $\varepsilon$.

We say that $x \in X$ generates a measure $\mu \in \mathcal{M}_{1}$ if $\mu \in \operatorname{clconv}\left\{Q^{t} \delta_{x}: t \geq 0\right\}$, where the closure is taken in the weak topology.

Proposition 1 Assume that $\left(P_{t}\right)_{t \geq 0}$ has the e-property. If $\mu \in \mathcal{M}_{1}$ is generated by $x \in$ $\mathcal{T}$, then the sequence $\left(Q^{t} \mu\right)_{t \geq 0}$ has the same limit as $\left(Q^{t}(x, \cdot)\right)_{t \geq 0}$. 
Proof Fix $f \in \mathcal{L}_{b}(X)$ with $\|f\| \leq 1$. Lemma 2 in [14] and the e-property give

$$
\lim _{n \rightarrow \infty} \sup _{t \geq 0}\left|\int_{X} f d Q^{t} \mu_{n}-\int_{X} f d Q^{t} \mu\right|=0
$$

provided $\mu_{n}$ weakly converges to $\mu$.

Fix $\varepsilon>0$ and let $\mu \in \mathcal{M}_{1}$ be generated by $x$. Then we may find $N \in \mathbb{N}$, $\alpha_{1}, \ldots, \alpha_{N} \geq 0$ and $v_{1}, \ldots, v_{N} \in \mathcal{M}_{1}$ such that $\alpha_{1}+\ldots+\alpha_{N}=1$ and

$$
\sup _{t \geq 0}\left|\int_{X} f d Q^{t}\left(\alpha_{1} Q^{t_{1}}(x, \cdot)+\ldots+\alpha_{N} Q^{t_{N}}(x, \cdot)\right)-\int_{X} f d Q^{t} \mu\right|<\varepsilon / 2
$$

for some $t_{1}, \ldots, t_{N} \geq 0$.

Due to Lemma 2 in [5] we have

$$
\left\|Q^{t} Q^{t_{i}}(x, \cdot)-Q^{t}(x, \cdot)\right\|_{T V}<\varepsilon / 2,
$$

for all $t$ large enough, say, $t \geq T$, and $i=1, \ldots, N$. Therefore,

$$
\begin{aligned}
& \left|\int_{X} f \mathrm{~d} Q^{t} \mu-\int_{X} f \mathrm{~d} Q^{t}(x, \cdot)\right| \\
& \leq\left|\int_{X} f d Q^{t} \mu-\int_{X} f d Q^{t}\left(\alpha_{1} Q^{t_{1}}(x, \cdot)+\ldots+\alpha_{N} Q^{t_{N}}(x, \cdot)\right)\right| \\
& \quad+\sum_{i=1}^{N} \alpha_{i}\left\|Q^{t} Q^{t_{i}}(x, \cdot)-Q^{t}(x, \cdot)\right\|_{T V}<\varepsilon
\end{aligned}
$$

for $t \geq T$. Consequently, if one of the sequences $\left(Q^{t} \mu\right)_{t \geq 0},\left(Q^{t}(x, \cdot)\right)_{t \geq 0}$ converges, then so does the second and both have the same limit. Lemma 1 finishes the proof.

Remark 1 Assume that $\left(P_{t}\right)_{t \geq 0}$ has the e-property. A measure $\mu$ is generated by $x$ iff $\mu \in \operatorname{cl} \operatorname{conv}\left\{Q^{t} v: v \in \mathcal{M}_{1}, \operatorname{supp} v \subset B(x, \varepsilon), t \geq 0\right\}$ for every $\varepsilon>0$.

Proof Applying the fact that for a given $\varepsilon>0$ and $f \in \mathcal{L}_{b}(X)$ there exists $\eta>0$ such that $\left|\int_{X} f \mathrm{~d} Q^{t} v-\int_{X} f \mathrm{~d} Q^{t}(x, \cdot)\right|<\varepsilon$ for $t \geq 0$ and any $v \in \mathcal{M}_{1}$ with supp $v \subset B(x, \eta)$, we can easily obtain the desired result.

Proposition 2 Assume that $\left(P_{t}\right)_{t \geq 0}$ has the e-property. If $\mu$ is an ergodic invariant measure, then every $x \in \operatorname{supp} \mu$ generates the measure $\mu$.

Proof The proof will be split into three steps.

Step I Fix $x \in \operatorname{supp} \mu$ and let $A \subset X$ be an open neighbourhood of $x$. Define

$$
B=\left\{y \in X: \pi^{t}(y, A)=0 \text { for all } t \geq 0\right\} .
$$

Since the semigroup $\left(P_{t}\right)_{t \geq 0}$ is stochastically continuous we easily check that

$$
B=\bigcap_{q \in \mathbb{Q}_{+}}\left\{y \in X: \pi^{q}(y, A)=0\right\} .
$$


Now we show that $\mu(B)=0$. Assume, contrary to our claim, that $\mu(B)>$ 0 . We shall prove that $P_{t} \mathbf{1}_{B}=\mathbf{1}_{B}$, hence $\mu(B)=1$, by the ergodicity of the measure $\mu$. On the other hand, since $B \subset X \backslash A$ and $\mu(A)>0$, this leads to a contradiction. So, to finish this step we show that $P_{t} \mathbf{1}_{\mathbf{B}} \geq$ 1. Fix $z \in B$. We have $P_{t} \mathbf{1}_{B}(z)=\int_{X} \mathbf{1}_{B}(y) \pi^{t}(z, \mathrm{~d} y)=\pi^{t}(z, B)$. Let $\mathbb{Q}_{+}=\left\{q_{1}, q_{2}, \ldots\right\}$. Set $C_{n}=\left\{y: \pi^{q_{n}}(y, A)>0\right\}$. Observe that if $\pi^{t}(z, X \backslash$ $B)>0$, then $\pi^{t}\left(z, C_{m}\right)>0$ for some $m \in \mathbb{N}$, since $X \backslash B=\bigcup_{i=1}^{\infty} C_{i}$. Then $\pi^{t+q_{m}}(z, A)=\int_{X} \pi^{q_{m}}(y, A) \pi^{t}(z, \mathrm{~d} y) \geq \int_{C_{m}} \pi^{q_{m}}(y, A) \pi^{t}(z, \mathrm{~d} y)>0$, contrary to our definition of $z$. Therefore $\mu(B)=1$ and we are done.

Step II Fix an $\varepsilon>0$. In this step we are going to show that

$$
\gamma:=\sup v(X)=1
$$

where the supremum is taken over all $v$ 's such that $\mu \geq v$ and $v=$ $\alpha_{1} P_{t_{1}}^{*} v_{1}+\ldots+\alpha_{n} P_{t_{n}}^{*} v_{n}$ for some probability measures $v_{1}, \ldots, v_{n}$ supported on $B(x, \varepsilon)$ and $\alpha_{1}, \ldots, \alpha_{n}, t_{1}, \ldots, t_{n} \geq 0$.

If not, there exist a sequence $\left(v_{n}\right)_{n \geq 1}$ such that $1>\gamma=\lim _{n \rightarrow \infty} v_{n}(X)$ and $v_{n}$ are as above. Set $\mu_{n}=\mu-v_{n}$ for $n \geq 1$. Obviously the sequence $\left(\mu_{n}\right)_{n \geq 1}$ is tight, and therefore there exists $\mu_{*} \neq 0$ such that $\mu_{n}$ 's converge weakly to $\mu_{*}$, passing to a subsequence if necessary. Let $A=B(x, \varepsilon)$. By Step I we may choose $z \in \operatorname{supp} \mu_{*} \subset \operatorname{supp} \mu$ and $t \geq 0$ such that $\eta=\pi^{t}(z, A)>$ 0 . From the Feller property it follows that there exists $\theta>0$ such that $\pi^{t}(y, A) \geq \eta / 2$ for any $y \in B(z, \theta)$. Denote by $\alpha=\mu_{*}(B(z, \theta))$. Let $N \in \mathbb{N}$ be such that $\gamma-v_{N}(X)<\eta \alpha / 4$ and $\mu_{N}(B(z, \theta))>\alpha / 2$ by the fact that $\left(v_{n}\right)_{n \geq 1}$ converges weakly to $\mu_{*}$ and by the Alexandrov theorem. Then we have

$$
P_{t}^{*} \mu_{N}(A)=\int_{X} \pi^{t}(y, A) \mu_{N}(\mathrm{~d} y) \geq \alpha \eta / 4
$$

and consequently we obtain

$$
\mu=P_{t}^{*} \mu \geq P_{t}^{*} v_{N}+(\alpha \eta / 4) \tilde{v},
$$

where $\tilde{v}(\cdot)=\left(P_{t}^{*} \mu-P_{t}^{*} v_{N}\right)(\cdot \cap A) /\left(P_{t}^{*} \mu-P_{t}^{*} v_{N}\right)(A)$. Hence $\gamma \geq v_{N}(X)+$ $\alpha \eta / 4$, which contradicts the definition of $v_{N}$.

Step III From the previous step it easily follows that $\mu$ is generated by any $x \in$ $\operatorname{supp} \mu$. Indeed, we see that for an arbitrary $\varepsilon>0$ we may find $v_{1}, \ldots, v_{N}$ supported on $B(x, \varepsilon)$ and $\alpha_{1}, \ldots, \alpha_{N} \geq 0, t_{1}, \ldots, t_{N} \geq 0$ such that

$$
\mu \geq \alpha_{1} P_{t_{1}}^{*} v_{1}+\ldots+\alpha_{N} P_{t_{N}}^{*} v_{N}
$$

and $\alpha_{1}+\ldots+\alpha_{N}>1-\varepsilon / 2$. Obviously we may choose $T>0$ such that

$$
\left\|Q^{T} v_{i}-Q^{T}\left(P_{t_{i}}^{*} v_{i}\right)\right\|_{T V}<\varepsilon / 2 \quad \text { for all } i \in\{1, \ldots, N\}
$$


Hence we obtain

$$
\begin{aligned}
\| \mu & -\left(\alpha_{1} Q^{T} v_{1}+\ldots+\alpha_{N} Q^{T} v_{N}\right) \|_{T V} \\
= & \left\|Q^{T} \mu-\left(\alpha_{1} Q^{T} v_{1}+\ldots+\alpha_{N} Q^{T} v_{N}\right)\right\|_{T V} \\
\leq & \left\|Q^{T} \mu-Q^{T}\left(\alpha_{1} P_{t_{1}}^{*} v_{1}+\ldots+\alpha_{N} P_{t_{N}}^{*} v_{N}\right)\right\|_{T V} \\
& +\left\|\left(\alpha_{1} Q^{T} P_{t_{1}}^{*} v_{1}+\ldots+\alpha_{N} Q^{T} P_{t_{N}}^{*} v_{N}\right)-\left(\alpha_{1} Q^{T} v_{1}+\ldots \alpha_{N} Q^{T} v_{N}\right)\right\|_{T V} \\
\leq & \left\|\mu-\left(\alpha_{1} P_{t_{1}}^{*} v_{1}+\ldots+\alpha_{N} P_{t_{N}}^{*} v_{N}\right)\right\|_{T V} \\
& +\sum_{i=1}^{N} \alpha_{i}\left\|Q^{T} v_{i}-Q^{T} P_{t_{i}}^{*} v_{i}\right\|_{T V}<\varepsilon .
\end{aligned}
$$

The use of Remark 1 ends the proof.

Theorem 1 Let $\left(P_{t}\right)_{t \geq 0}$ be a Markov semigroup on a Polish space $X$ admitting two distinct ergodic invariant measures $\mu$ and $\nu$. If $\left(P_{t}\right)_{t \geq 0}$ has the e-property, then supp $\mu \cap$ $\operatorname{supp} v=\emptyset$.

Proof Let $x \in \operatorname{supp} \mu \cap \operatorname{supp} v$. Due to Proposition 1 in [5] $x$ belongs to $\mathcal{T}$. By Proposition 2 we obtain that $\mu$ and $v$ are generated by $x$. On the other hand, from Proposition 1 both the measures $\mu$ and $v$ are the weak limit of the sequence $\left(Q^{t}(x, \cdot)\right)_{t \geq 0}$ and therefore $\mu=v$.

We say that a Markov semigroup $\left(P_{t}\right)_{t \geq 0}$ is weakly topologically irreducible if for all $x_{1}, x_{2} \in X$ there exists $y \in X$ such that for any open set $A \ni y$ there exist $t_{1}, t_{2}>0$ with $\pi^{t_{i}}\left(x_{i}, A\right)>0$ for $i=1,2$.

Theorem 2 Let $\left(P_{t}\right)_{t \geq 0}$ be weakly topologically irreducible. If $\left(P_{t}\right)_{t \geq 0}$ has the $e$ property, then it has at most one invariant measure.

Proof The proof consists in observing that if there exist at least two different invariant measures, then not less than two different ergodic invariant measures exist. Topological irreducibility and the Feller property give that their supports do intersect. The immediate application of Theorem 1 finishes the proof.

\section{Applications to SPDE's}

Let $H$ be a Hilbert space with some scalar product $\langle\cdot, \cdot\rangle$. Let $\left\{Y_{x}(t)\right\}_{t \geq 0}, x \in H$, be a unique mild solution to the equation

$$
\mathrm{d} Y=(A Y+F(Y)) \mathrm{d} t+\mathrm{d} Z(t) \quad \text { for } t \geq 0 \text { with } Y_{x}(0)=x,
$$

where $Z$ is some noise on $H$.

We will additionally assume that the process $\{Y(t)\}_{t \geq 0}$ satisfies the Feller property.

Recall that a map $G: H \rightarrow H$ is called weakly dissipative if

$$
\langle G(x)-G(y), x-y\rangle \leq 0 \quad \text { for all } x, y \in H .
$$


The following easy lemma provides us with a sufficient condition for the eproperty.

Lemma 2 If the mapping $G(Y)=A Y+F(Y)$ is weakly dissipative, then the process $\{Y(t)\}_{t \geq 0}$ has the e-property.

Proof Let $Y_{x}$ and $Y_{y}$ be two solutions to Eq. 3.1 with the initial conditions $Y(0)=x$ and $Y(0)=y$, respectively. Since

$$
\mathrm{d}\left(Y_{x}-Y_{y}\right)=\left(A\left(Y_{x}-Y_{y}\right)+F\left(Y_{x}\right)-F\left(Y_{y}\right)\right) \mathrm{d} t,
$$

we have

$$
\frac{1}{2} \frac{\mathrm{d}}{\mathrm{d} t} \mathbb{E}\left\|Y_{x}-Y_{y}\right\|^{2} \leq \mathbb{E}\left(\left\langle G\left(Y_{x}\right)-G\left(Y_{y}\right), Y_{x}-Y_{y}\right\rangle\right) \leq 0,
$$

by dissipativeness of $G$. Consequently $\mathbb{E}\left\|Y_{x}-Y_{y}\right\|^{2} \leq\|x-y\|^{2}$.

Let $f: H \rightarrow \mathbb{R}$ be an arbitrary Lipschitz function. Denote by $L_{f}$ its Lipschitz constant. Then using the Cauchy-Schwartz inequality we have

$$
\begin{aligned}
\left|P_{t} f(x)-P_{t} f(y)\right| & \leq \mathbb{E}\left|f\left(Y_{x}(t)\right)-f\left(Y_{y}(t)\right)\right| \\
& \leq L\left(\mathbb{E}\left\|\left(Y_{x}(t)-Y_{y}(t) \|^{2}\right)^{1 / 2} \leq L_{f}\right\| x-y \|,\right.
\end{aligned}
$$

which finishes the proof.

As a consequence of Theorem 2 we have the following proposition.

Proposition 3 If the semigroup $\left(P_{t}\right)_{t \geq 0}$ corresponding to Eq. 3.1 is weakly irreducible and the deterministic part of the equation is weakly dissipative, then there exists at most one invariant measure.

The above result seems to be a useful tool in studing ergodicity of weakly dissipative systems disturbed with some additive noise. We exemplify it by proving the uniqueness of an invariant measure for some stochastic heat equation driven by an impulsive noise. These equations were studied by Peszat and Zabczyk (see $[10,11])$. Another possible application of this result to weakly dissipative stochastic evolution equations will be provided in a subsequent paper.

Let $E=[0, \infty) \times[0,1] \times \mathbb{R}$. Define a Borel measure $\mu$ on $E$ by $\mu(\mathrm{d} t, \mathrm{~d} x, \mathrm{~d} \sigma)=$ $\mathrm{d} t \mathrm{~d} x v(\mathrm{~d} \sigma)$, where $v$ is a finite Borel measure on $\mathbb{R}$ with supp $v=\mathbb{R}$. We suppose that $\left\{U_{n}\right\}$ is a disjoint partition of $\mathbb{R} \backslash\{0\}$ such that

$$
\int_{U_{n}} \sigma v(d \sigma)=0
$$

We assume that

$$
a_{v}=\int_{\mathbb{R}} \sigma^{2} v(d \sigma)<\infty .
$$


Let $\left\{O_{n}\right\}$ be a disjoint partition of $[0,1]$. Let $\left(\xi_{j}^{(n, m)}\right),\left(x_{j}^{(n, m)}\right)$ and $\left(\sigma_{j}^{(n, m)}\right), n, m \in \mathbb{N}$, be independent random elements defined on some probability space $(\Omega, \mathcal{F}, \mathbb{P})$ and taking values in $[0, \infty),[0,1]$, and $\mathbb{R}$, respectively such that

$$
\begin{array}{cl}
\mathbb{P}\left(\xi_{j}^{(n, m)}>t\right)=e^{-\kappa_{n, m} t} & \text { for } t \geq 0, \\
\mathbb{P}\left(x_{j}^{(n, m)} \in B\right)=\frac{\left|B \cap O_{m}\right|}{\left|O_{m}\right|} \quad \text { for } B \in \mathcal{B}([0,1]), \\
\mathbb{P}\left(\sigma_{j}^{(n, m)} \in A\right)=\frac{v\left(A \cap U_{n}\right)}{v\left(U_{n}\right)} \quad \text { for } A \in \mathcal{B}(\mathbb{R}),
\end{array}
$$

where $\kappa_{n, m}=v\left(U_{n}\right)\left|O_{m}\right|$ and $|\cdot|$ denotes the Lebesgue measure on $\mathbb{R}$.

Set $\tau_{k}^{(n, m)}=\xi_{1}^{(n, m)}+\ldots+\xi_{k}^{(n, m)}$. We will consider the measure valued process of the form

$$
Z(t, d x)=\sum_{\tau_{j}^{(n, m)} \leq t} \sigma_{j}^{(n, m)} \delta_{x_{j}^{(n, m)}}(d x) .
$$

This process is called the impulsive white noise (see [10]). The random variables $\sigma_{j}^{(n, m)}, \tau_{j}^{(n, m)}$ and $x_{j}^{(n, m)}$ are interpreted as an amount of energy introduced to the system, the random moment and the random place it happens, respectively.

Let $\left(Z_{k}\right)_{k \geq 1}$ be independent copies of the measure valued process $Z$ defined on a given probability space $(\Omega, \mathcal{F}, \mathbb{P})$.

Now we are in a position to recall the definition of the stochastic integral with respect to the measure valued process $Z$. Let $\mathcal{F}_{t}^{\mathcal{Z}}=\sigma\left\{Z_{k}(s, A): 0 \leq s \leq t, A \in\right.$ $\mathcal{B}([0,1]), k \geq 1\}$ and let $\mathcal{F}_{t}:=\overline{\mathcal{F}_{t+}^{\mathcal{Z}}}$, where $\overline{\mathcal{F}_{t+}^{\mathcal{Z}}}$ is the completion of $\mathcal{F}_{t+}^{\mathcal{Z}}$ with respect to the measure $\mathbb{P}$. First we define the stochastic integral of a random field of the form

$$
X(\omega, s, \cdot)=\xi(\omega) \mathbf{1}_{(a, b]}(s) \mathbf{1}_{A}(\cdot),
$$

where $\xi$ is a bounded and $\mathcal{F}_{a}$-measurable random variable. We set

$$
\int_{0}^{t} X(s) \mathrm{d} Z_{k}(s)=\left(Z_{k}(b \wedge t, A)-Z_{k}(a \wedge t, A)\right) \xi \quad \text { for any } k \geq 1 .
$$

Further, for any Hilbert space $H$ we define

$$
\mathcal{P}_{T, Z}(H)=\mathbb{L}^{2}\left(\Omega \times[0, T], \mathcal{B}_{T}, \mathrm{~d} \mathbb{P} \otimes \mathrm{d} t ; H\right),
$$

where $\mathcal{B}_{T}$ denotes the predictable $\sigma$-field on $\Omega \times[0, T]$. More precisely, $\mathcal{B}_{T}$ is generated by products $\xi \mathbf{1}_{(a, b]}$, where $0 \leq a<b<T$ and the $H$-valued random variable $\xi$ is $\mathcal{F}_{a}$-measurable. It can be proved that the space of all linear combinations of the form (3.3) is dense in $\mathcal{P}_{T, Z}\left(\mathbb{L}^{2}\right)$. (Here $\mathbb{L}^{2}$ denotes the space of all Borel measurable functions defined on $[0,1]$ which are square-integrable with respect to Lebesgue measure. By $\|\cdot\|$ we will denote the norm in $\mathbb{L}^{2}$.)

We can easily show that

$$
\mathbb{E}\left|\int_{t_{1}}^{t_{2}} X(s) \mathrm{d} Z_{k}(s)\right|^{2}=a_{v} \mathbb{E} \int_{t_{1}}^{t_{2}}\|X(s)\|^{2} \mathrm{~d} s
$$


for $0 \leq t_{1} \leq t_{2}<T, k \geq 1$ and any random field of the form (3.3). Since such random fields are dense in $\mathcal{P}_{T, Z}\left(\mathbb{L}^{2}\right)$, condition (3.4) holds for any $X \in \mathcal{P}_{T, Z}\left(\mathbb{L}^{2}\right)$.

As usual for two separable Hilbert spaces with complete orthonormal bases $\left\{e_{n}\right\} \subset$ $E,\left\{f_{n}\right\} \subset F$ and norms $|\cdot|_{E},|\cdot|_{F}$, respectively, by $L_{(H, S)}(E, F)$ we denote the space of all Hilbert-Schmidt operators, i.e. linear bounded operators $T: E \rightarrow F$ such that

$$
\|T\|_{(H, S)}:=\left(\sum_{n \geq 1}\left|T e_{n}\right|_{F}^{2}\right)^{1 / 2}<\infty
$$

Finally, for $X \in \mathcal{P}_{T, Z}\left(L_{(H, S)}\left(\mathbb{L}^{2}, \mathbb{L}^{2}\right)\right)$ we define

$$
\int_{t_{1}}^{t_{2}} X(s) d \mathcal{Z}(s)=\sum_{n \geq 1} \int_{t_{1}}^{t_{2}}\left(X(s)^{*} e_{n}\right) \mathrm{d} Z_{n}(s) e_{n}
$$

where $\left\{e_{n}\right\}$ is the orthonormal basis of $\mathbb{L}^{2}$ of the form $e_{n}(x)=\sqrt{2} \sin (\pi n x)$ for $x \in[0,1]$ and $n \in \mathbb{N}$. To show that the above definition is accurate observe that the series $\sum_{n \geq 1} \int_{0}^{t}\left(X(s)^{*} e_{n}\right) \mathrm{d} Z_{n}(s) e_{n}$ for arbitrary $t \geq 0$ is convergent in $\mathbb{L}^{2}\left(\Omega, \mathcal{F}_{t}, \mathbb{P} ; \mathbb{L}^{2}\right)$. Namely, by Eq. 3.4 we have

$$
\sum_{n \geq 1} \mathbb{E}\left(\int_{0}^{t}\left(X(s)^{*} e_{n}\right) \mathrm{d} Z_{n}(s)\right)^{2}=a_{v} \mathbb{E}\left(\sum_{n \geq 1} \int_{0}^{t}\left\|X(s)^{*} e_{n}\right\|^{2} \mathrm{~d} s\right)=a_{v} \mathbb{E} \int_{0}^{t}\|X(s)\|_{(H, S)}^{2} \mathrm{~d} s .
$$

Obviously, for any $X \in \mathcal{P}_{T, Z}\left(L_{(H, S)}\left(\mathbb{L}^{2}, \mathbb{L}^{2}\right)\right)$ the stochastic integral $\int_{0}^{t} X(s) \mathrm{d} \mathcal{Z}(s)$, $t \in[0, T]$ is a square integrable $\mathbb{L}^{2}$ martingal. By Eq. 3.4 it is also stochastically continuous and hence by Doob's theorem it has a càdlàg modification.

Consider the stochastic heat equation

$$
\begin{array}{cc}
\frac{\partial u}{\partial t}(t, x) & =\Delta u(t, x)-g(u(t, x))+\frac{\partial \mathcal{Z}}{\partial t}(t, x) \\
u(t, 0) & =u(t, 1)=0 \quad \text { for } t>0
\end{array}
$$

with the initial condition

$$
\left.u\right|_{t=0}=\xi,
$$

where $\xi$ is measurable with respect to $\mathcal{F}_{0}$.

In what follows we assume that $g: \mathbb{R} \rightarrow \mathbb{R}$ is continuously differentiable with bounded derivative $g^{\prime}$ and its Lipschitz constant is denoted by $L_{g}$.

By a solution we will understand the so-called mild solution, that is a predictable process taking values in $\mathbb{L}^{2}$ such that for every $t$

$$
u_{\xi}(t)=S(t) \xi+\int_{0}^{t} S(t-s) g\left(u_{\xi}(s)\right) d s+\int_{0}^{t} S(t-s) d \mathcal{Z}(s)
$$

where $(S(t))_{t \geq 0}$ is the semigroup generated by the Laplace operator with the Dirichlet boundary conditions. It has the following representation

$$
S(t) \phi=\sum_{k \geq 1} e^{-\pi^{2} k^{2} t}\left\langle\phi, e_{k}\right\rangle e_{k},
$$


where $e_{k}(x)=\sqrt{2} \sin (\pi k x), k \in \mathbb{N}, x \in[0,1]$ and $\langle\cdot, \cdot\rangle$ denotes the standard scalar product in $\mathbb{L}^{2}$.

The proof of the existence and uniqueness of the solution to the above stochastic heat equation is standard. It parallels mostly the proof of Theorem 3.1 in [10] and therefore we omit it here. From the mentioned paper it follows that $\left(P_{t}\right)_{t \geq 0}$ given by the formula

$$
P_{t} f(x)=\mathbb{E} f\left(u_{x}(t)\right) \quad \text { for } f \in C\left(\mathbb{L}^{2}\right) \text { and } t \geq 0,
$$

where $u_{x}$ denotes the solution with $\xi \equiv x$, defines a Feller semigroup.

We are aimed at proving the uniqueness of an invariant measure under the assumption that $L_{g} \leq \pi^{2}$. It is worth mentionig here that the novelty of our result concerns the case $L_{g}=\pi^{2}$. If $L_{g}<\pi^{2}$, then the system is strongly dissipative and it admits a unique attractive invariant meassure.

We start with a rather technical lemma useful in proving irreducibility of the solutions to our equation.

Lemma 3 Let $X \in \mathbb{L}^{2}\left([0, T]: \mathbb{L}^{2}\right)$ be such that $\int_{s}^{t} \int_{0}^{1}|X(u)(x)| \mathrm{d} x \mathrm{~d} u>0$. Then for any $a \in \mathbb{R}$ and $\varepsilon>0$ we have

$$
\mathbb{P}\left(\left|\int_{s}^{t} X(u) \mathrm{d} Z(u)-a\right|<\varepsilon \text { and } \sup _{s \leq \tau \leq t}\left|\int_{s}^{\tau} X(u) \mathrm{d} Z(u)\right|<|a|+\varepsilon\right)>0 .
$$

Proof Fix $a \in \mathbb{R}$. There is no loss of generality in assuming that $a \neq 0$. We may also assume that $s=0$. Fix an $\varepsilon>0$. Since $\int_{0}^{t} \int_{0}^{1}|X(u)(x)| \mathrm{d} x \mathrm{~d} u \neq 0$, there exists $m \geq 1$ such that

$$
\int_{0}^{t} \int_{O_{m}} X(u)(x) \mathrm{d} x \mathrm{~d} u \neq 0 .
$$

From Eq. 3.10 it follows that there exists $v \in \mathbb{R} \backslash\{0\}$ and $\Lambda \subset[0, t] \times O_{m}$ with positive Lebesgue measure $\mathcal{L}_{2}$ such that

$$
|X(u)(x)-v|<\varepsilon|v| /(3|a|) \quad \text { for }(u, x) \in \Lambda .
$$

Set $\gamma=a / v$ and let $n \geq 1$ be such that $\gamma \in U_{n}$. Choose $\eta \in(0, \min \{\varepsilon /(3|v|),|a / v|\})$. Set

$$
\begin{aligned}
& \Omega_{1}=\left\{\omega \in \Omega: \xi_{1}^{(k, l)}(\omega)>t \text { for al }(k, l) \in \mathbb{N} \times \mathbb{N} \backslash\{(n, m)\}\right\}, \\
& \Omega_{2}=\left\{\omega \in \Omega:\left(\xi_{1}^{(n, m)}(\omega), x_{1}^{(n, m)}(\omega)\right) \in \Lambda\right\}, \\
& \Omega_{3}=\left\{\omega \in \Omega: \xi_{2}^{(n, m)}(\omega)>t\right\}
\end{aligned}
$$

and

$$
\Omega_{4}=\left\{\omega \in \Omega: \sigma_{1}^{(n, m)}(\omega) \in(\gamma-\eta, \gamma+\eta)\right\} .
$$

Let $\Omega_{0}=\bigcap_{i=1}^{4} \Omega_{i}$. First observe that the sets $\Omega_{1}, \Omega_{2}, \Omega_{3}, \Omega_{4}$ are independent. Indeed, it follows from the fact that $\left(\xi_{j}^{(k, l)}\right),\left(x_{j}^{(k, l)}\right)$ and $\left(\sigma_{j}^{(k, l)}\right)$ for $k, l, j \in \mathbb{N}$ are independent. Further, by the definition of $\gamma$ and $\eta$ we easily check that 


$$
\left|\int_{0}^{t} X(u)(x) \mathrm{d} Z(u)(\omega)-a\right|<\varepsilon
$$

and

$$
\left|\int_{0}^{\tau} X(u)(x) \mathrm{d} Z(u)(\omega)\right|<|a|+\varepsilon \quad \text { for any } \omega \in \Omega_{0} \text { and } \tau \in[0, t] .
$$

Since the set $\Omega_{1}, \Omega_{2}, \Omega_{3}, \Omega_{4}$ are independent, to prove that $\mathbb{P}\left(\Omega_{0}\right)>0$ it is enough to show that $\mathbb{P}\left(\Omega_{i}\right)>0$ for $i=1,2,3,4$. Obviously the sets $\Omega_{3}, \Omega_{4}$ have positive measure, the last by the fact that $\operatorname{supp} v \cap(\gamma-\eta, \gamma+\eta) \neq \emptyset$. Since $\mathcal{L}_{2}(\Lambda)>0$, the distribution $\Phi_{\left(\xi_{1}^{(n, m)}, x_{1}^{(n, m)}\right)}$ of the vector $\left(\xi_{1}^{(n, m)}, x_{1}^{(n, m)}\right)$ is absolutely continuous with respect to $\mathcal{L}_{2}$ and its density is equal to $\kappa_{n, m} e^{-\kappa_{n, m} t}\left|O_{m}\right|^{-1} \mathbf{1}_{[0,+\infty) \times O_{m}}(t, x)$, we obtain that $\mathbb{P}\left(\Omega_{2}\right)>0$. To finish the proof we have to show that $\mathbb{P}\left(\Omega_{1}\right)>0$. But we have

$$
\mathbb{P}\left(\Omega_{1}\right)=\prod_{(k, l) \neq(n, m)} \mathbb{P}\left(\xi_{1}^{(k, l)}>t\right)=\exp \left\{-t \sum_{(k, l) \neq(n, m)} v\left(U_{k}\right)\left|O_{l}\right|\right\} \geq e^{-v(\mathbb{R}) t}>0 .
$$

The proof is complete.

Denote by $\mathcal{Z}_{S}(t)$ the convolution $\int_{0}^{t} S(t-s) \mathrm{d} \mathcal{Z}(s)$. We have the following.

Lemma 4 Let $f \in \mathbb{L}^{2}\left([0, T]: \mathbb{L}^{2}\right)$ and $g \in \mathbb{L}^{2}$. Then for any $\varepsilon>0$ we have

$$
\mathbb{P}\left(\int_{0}^{T}\left\|f(t)-\mathcal{Z}_{S}(t)\right\|^{2} \mathrm{~d} t<\varepsilon \text { and }\left\|\mathcal{Z}_{S}(T)-g\right\|<\varepsilon\right)>0 .
$$

Proof Fix $f \in \mathbb{L}^{2}\left([0, T]: \mathbb{L}^{2}\right)$ and $g \in \mathbb{L}^{2}$. Without loss of generality we may assume that

$$
f=\sum_{j=1}^{N} f_{j} \mathbf{1}_{\left[t_{j-1}, t_{j}\right)}
$$

where $0=t_{0}<t_{1}<\ldots<t_{N}=T$ and $f_{1}, \ldots, f_{N} \in \mathbb{L}^{2}$ for some $N \in \mathbb{N}$. We may also assume that $f_{1} \equiv 0$ and $f_{N}=g$. Finally, we shall assume that $\left\langle f_{j}, e_{m}\right\rangle=0$ for $j \in$ $\{1, \ldots, N\}$ and all $m \geq m_{0}$ for sufficiently large $m_{0}$.

Since

$$
\mathbb{E} \int_{0}^{T}\left\|\mathcal{Z}_{S}(t)\right\|^{2} \mathrm{~d} t=\sum_{k \geq 1} \mathbb{E} \int_{0}^{T}\left\langle\mathcal{Z}_{S}(t), e_{k}\right\rangle^{2} \mathrm{~d} t<\infty
$$

we obtain that

$$
\lim _{n \rightarrow \infty} \mathbb{E}\left(\sum_{k \geq n} \int_{0}^{T}\left\langle\mathcal{Z}_{S}(t), e_{k}\right\rangle^{2} \mathrm{~d} t\right)=0
$$

and consequently

$$
\mathbb{P}\left(\sum_{k \geq n} \int_{0}^{T}\left\langle\mathcal{Z}_{S}(t), e_{k}\right\rangle^{2} \mathrm{~d} t<\varepsilon\right)>0
$$


for any $\varepsilon>0$ and $n$ depending upon $\varepsilon$. Thus to finish the proof of lemma it is enough to show that for every $i \in \mathbb{N}$ and $\varepsilon>0$

$$
\mathbb{P}\left(\int_{0}^{T}\left\langle\mathcal{Z}_{S}(t)-f, e_{i}\right\rangle^{2} \mathrm{~d} t<\varepsilon \text { and }\left|\left\langle\mathcal{Z}_{S}(T)-f_{N}(T), e_{i}\right\rangle\right|<\varepsilon\right)>0 .
$$

Indeed, our statement follows then from the fact that the events

$$
\left\{\omega \in \Omega: \int_{0}^{T}\left\langle\mathcal{Z}_{S}(t)(\omega)-f, e_{i}\right\rangle^{2} \mathrm{~d} t<\varepsilon \text { and }\left|\left\langle\mathcal{Z}_{S}(T)(\omega)-f_{N}(T), e_{i}\right\rangle\right|<\varepsilon\right\}
$$

for $i<n$ and

$$
\left\{\omega \in \Omega: \sum_{k \geq n} \int_{0}^{T}\left\langle\mathcal{Z}_{S}(t)(\omega), e_{k}\right\rangle^{2} \mathrm{~d} t<\varepsilon\right\}
$$

are independent for any $\varepsilon>0$ and $n \in \mathbb{N}$.

Fix $\varepsilon>0$ and $i \in \mathbb{N}$. Set $\tilde{\varepsilon}=\varepsilon / T$ and $\delta=\max _{1 \leq j \leq N}\left(t_{j}-t_{j-1}\right)$. Splitting, if necessary, the intervals $\left[t_{j-1}, t_{j}\right]$ into smaller subintervals, we may assume that

$$
\max _{1 \leq j \leq N}\left|\left(1-e^{-\pi^{2} i^{2} \delta}\right)\left\langle f_{j}, e_{i}\right\rangle\right|<\tilde{\varepsilon} / 8 .
$$

To verify Eq. 3.11 we shall prove by induction that for any $j \in\{0, \ldots, N\}$ we have

$$
\mathbb{P}\left(\int_{0}^{t_{j}}\left\langle\mathcal{Z}_{S}(t)-f, e_{i}\right\rangle^{2} \mathrm{~d} t \leq \tilde{\varepsilon} t_{j} \text { and }\left|\left\langle\mathcal{Z}_{S}\left(t_{j}\right)-f\left(t_{j}\right), e_{i}\right\rangle\right| \leq \tilde{\varepsilon}\right)>0 .
$$

Our hypothesis is obviously satisfied for $j=0$. Assume now that it holds for some $l<N$. We shall show that it holds for $l+1$ as well. Put

$$
\Omega_{l}=\left\{\omega \in \Omega: \int_{0}^{t_{l}}\left\langle\mathcal{Z}_{S}(t)(\omega)-f, e_{i}\right\rangle^{2} \mathrm{~d} t \leq \tilde{\varepsilon} t_{l} \text { and }\left|\left\langle\mathcal{Z}_{S}\left(t_{l}\right)-f\left(t_{l}\right), e_{i}\right\rangle\right| \leq \tilde{\varepsilon}\right\} .
$$

Choose $\gamma \in \operatorname{supp} \mathcal{L}_{\Omega_{l}}\left(\left\langle\mathcal{Z}_{S}\left(t_{l}\right)(\cdot), e_{i}\right\rangle\right)$ where $\mathcal{L}_{\Omega_{l}}(X)$ stands for the law of a random element $X$ on the space $\left(\Omega_{l}, \mathcal{F}_{\Omega_{l}}, \mathbb{P}_{\Omega_{l}}\right)$. (Here $\mathcal{F}_{\Omega_{l}}=\left\{A \cap \Omega_{l}: A \in \mathcal{F}\right\}$ and $\mathbb{P}_{\Omega_{l}}(\cdot)=$ $\mathbb{P}\left(\cdot \mid \Omega_{l}\right)$.) Set $h=\left\langle f_{l+1}, e_{i}\right\rangle$ and let $\eta=h-\gamma$. Choose $s \in\left(t_{l}, t_{l+1}\right)$ small enough the following condition holds

$$
\left[(|\gamma|+\tilde{\varepsilon} / 8)^{2}+2(|l|+\tilde{\varepsilon} / 8)^{2}+h^{2}\right]\left(s-t_{l}\right) \leq \tilde{\varepsilon}\left(t_{l+1}-t_{l}\right) / 6 .
$$

Set

$$
\begin{array}{r}
\Omega_{l}^{1}=\left\{\omega \in \Omega:\left|\int_{t_{l}}^{s} e^{-\pi^{2} i^{2}\left(t_{l}-t\right)} e_{i} \mathrm{~d} Z_{i}(t)-\eta\right|<\tilde{\varepsilon} / 8\right. \\
\text { and } \left.\sup _{t_{l} \leq \tau \leq s}\left|\int_{t_{l}}^{\tau} e^{-\pi^{2} i^{2}\left(t_{l}-t\right)} e_{i} \mathrm{~d} Z_{i}(t)\right| \leq|\eta|+\tilde{\varepsilon} / 8\right\}
\end{array}
$$

and observe that $\mathbb{P}\left(\Omega_{l}^{1}\right)>0$, by Lemma 2 . Now, let

$$
\Omega_{l}^{2}=\left\{\omega \in \Omega_{l}:\left\langle\mathcal{Z}_{S}\left(t_{l}\right), e_{i}\right\rangle \in(\gamma-\tilde{\varepsilon} / 8, \gamma+\tilde{\varepsilon} / 8)\right\} .
$$

Obviously $\mathbb{P}\left(\Omega_{l}^{2}\right)>0$, by the definition of $\gamma$. Finally define

$$
\Omega_{l}^{3}=\left\{\omega \in \Omega: \sup _{s \leq \tau \leq t_{l+1}}\left|\int_{s}^{\tau} e^{-\pi^{2} i^{2}(s-t)} e_{i} \mathrm{~d} Z_{i}(t)\right| \leq \tilde{\varepsilon} / 8\right\}
$$


and observe that $\mathbb{P}\left(\Omega_{l}^{3}\right)>0$, by Lemma 2 . Define $\Omega_{l+1}^{0}=\Omega_{l+1}^{1} \cap \Omega_{l+1}^{2} \cap \Omega_{l+1}^{3}$. Since $\Omega_{l+1}^{1}, \Omega_{l+1}^{2}, \Omega_{l+1}^{3}$ are independent and all of them have positive measure, we have $\mathbb{P}\left(\Omega_{l+1}^{0}\right)>0$. Now let $\omega \in \Omega_{l+1}^{0}$. We check that

$$
\int_{0}^{t_{l+1}}\left\langle\mathcal{Z}_{S}(t)(\omega)-f, e_{i}\right\rangle^{2} \mathrm{~d} t \leq \tilde{\varepsilon} t_{l+1}
$$

and

$$
\left|\left\langle\mathcal{Z}_{S}\left(t_{l+1}\right)-f\left(t_{l+1}\right), e_{i}\right\rangle\right| \leq \tilde{\varepsilon} .
$$

By the induction assumption we have

$$
\begin{aligned}
\int_{0}^{t_{l+1}}\left\langle\mathcal{Z}_{S}(t)(\omega)-f, e_{i}\right\rangle^{2} \mathrm{~d} t= & \int_{0}^{t_{l}}\left\langle\mathcal{Z}_{S}(t)(\omega)-f, e_{i}\right\rangle^{2} \mathrm{~d} t+\int_{t_{l}}^{t_{l+1}}\left|\left\langle\mathcal{Z}_{S}(t)(\omega), e_{i}\right\rangle-h_{l+1}\right|^{2} \mathrm{~d} t \\
\leq & \tilde{\varepsilon} t+\int_{t_{l}}^{s}\left|\left\langle\mathcal{Z}_{S}(t)(\omega), e_{i}\right\rangle-h_{l+1}\right|^{2} \mathrm{~d} t \\
& +\int_{s}^{t_{l+1}}\left|\left\langle\mathcal{Z}_{S}(t)(\omega), e_{i}\right\rangle-h_{l+1}\right|^{2} \mathrm{~d} t .
\end{aligned}
$$

To evaluate the above integral set

$$
\mathbf{I}=\int_{t_{l}}^{s}\left|\left\langle\mathcal{Z}_{S}(t)(\omega), e_{i}\right\rangle-h_{l+1}\right|^{2} \mathrm{~d} t \text { and } \mathbf{I I}=\int_{t_{l}}^{t_{l+1}}\left|\left\langle\mathcal{Z}_{S}(t)(\omega), e_{i}\right\rangle-h_{l+1}\right|^{2} \mathrm{~d} t .
$$

Indeed, keeping in mind that

$$
\left\langle\mathcal{Z}_{S}(t)(\omega), e_{i}\right\rangle=\int_{0}^{t} e^{-\pi^{2} i^{2}(t-u)} e_{i} \mathrm{~d} Z_{i}(u),
$$

we have

$$
\begin{aligned}
\mathbf{I}= & \int_{t_{l}}^{s}\left|e^{-\pi^{2} i^{2}\left(t-t_{l}\right)}\left\langle\mathcal{Z}_{S}\left(t_{l}\right), e_{i}\right\rangle+e^{-\pi^{2} i^{2}\left(t-t_{l}\right)} \int_{t_{l}}^{t} e^{-\pi^{2} i^{2}\left(t_{l}-u\right)} e_{i} \mathrm{~d} Z_{i}(u)-h_{l+1}\right|^{2} \mathrm{~d} t \\
\leq & 3 \int_{t_{l}}^{s} e^{-2 \pi^{2} i^{2}\left(t-t_{l}\right)}\left\langle\mathcal{Z}_{S}\left(t_{l}\right), e_{i}\right\rangle^{2} \mathrm{~d} t+3 \int_{t_{l}}^{s} e^{-2 \pi^{2} i^{2}\left(t-t_{l}\right)}\left(\int_{t_{l}}^{t} e^{-\pi^{2} i^{2}\left(t_{l}-u\right)} e_{i} \mathrm{~d} Z_{i}(u)\right)^{2} \mathrm{~d} t \\
& +3 \int_{t_{l}}^{s} h_{l+1}^{2} \mathrm{~d} t .
\end{aligned}
$$

Further, observe that

$$
\int_{t_{l}}^{s} e^{-2 \pi^{2} i^{2}\left(t-t_{l}\right)}\left\langle\mathcal{Z}_{S}\left(t_{l}\right), e_{i}\right\rangle^{2} \mathrm{~d} t \leq(|\gamma|+\tilde{\varepsilon} / 8)^{2}\left(s-t_{l}\right),
$$

by the fact that $\omega \in \Omega_{l}^{2}$. On the other hand, since $\omega \in \Omega_{l}^{1}$ we have

$$
\int_{t_{l}}^{s} e^{-2 \pi^{2} i^{2}\left(t-t_{l}\right)}\left(\int_{t_{l}}^{t} e^{-\pi^{2} i^{2}\left(t_{l}-u\right)} e_{i} \mathrm{~d} Z_{i}(u)\right)^{2} \mathrm{~d} t \leq(|\eta|+\tilde{\varepsilon} / 8)^{2}\left(s-t_{l}\right)
$$

and consequently

$$
\mathbf{I} \leq 3\left[(|\gamma|+\tilde{\varepsilon} / 8)^{2}+2(|\eta|+\tilde{\varepsilon} / 8)^{2}+h_{l+1}^{2}\right]\left(s-t_{l}\right) \leq \tilde{\varepsilon}\left(t_{l+1}-t_{l}\right) / 2 .
$$


To evaluate integral II we shall prove that for $\omega \in \Omega_{l+1}^{0}$ we have

$$
\left|\left\langle\mathcal{Z}_{S}(t)-f(t), e_{i}\right\rangle\right|=\left|\left\langle\mathcal{Z}_{S}(t), e_{i}\right\rangle-h_{l+1}\right| \leq \tilde{\varepsilon} / 2 \quad \text { for } t \in\left[s, t_{l+1}\right] .
$$

To do this fix $t \in\left[s, t_{l+1}\right]$. Then

$$
\begin{aligned}
\left|\left\langle\mathcal{Z}_{S}(t), e_{i}\right\rangle-h_{l+1}\right|= & \left|\int_{0}^{s} e^{-\pi^{2} i^{2}(t-u)} e_{i} \mathrm{~d} Z_{i}(u)+\int_{s}^{t} e^{-\pi^{2} i^{2}(t-u)} e_{i} \mathrm{~d} Z_{i}(u)-h_{l+1}\right| \\
\leq & \left|\int_{s}^{t} e^{-\pi^{2} i^{2}(t-u)} e_{i} \mathrm{~d} Z_{i}(u)\right| \\
& +\left|\int_{0}^{t_{l}} e^{-\pi^{2} i^{2}(t-u)} e_{i} \mathrm{~d} Z_{i}(u)+\int_{t_{l}}^{s} e^{-\pi^{2} i^{2}(t-u)} e_{i} \mathrm{~d} Z_{i}(u)-h_{l+1}\right| \\
\leq & e^{-\pi^{2} i^{2}(t-s)}\left|\int_{s}^{t} e^{-\pi^{2} i^{2}(s-u)} e_{i} \mathrm{~d} Z_{i}(u)\right| \\
& +e^{-\pi^{2} i^{2}\left(t-t_{l}\right)}\left|\int_{0}^{t_{l}} e^{-\pi^{2} i^{2}\left(t_{l}-u\right)} e_{i} \mathrm{~d} Z_{i}(u)-\gamma\right| \\
& +e^{-\pi^{2} i^{2}\left(t-t_{l}\right)}\left|\int_{t_{l}}^{s} e^{-\pi^{2} i^{2}\left(t_{l}-u\right)} e_{i} \mathrm{~d} Z_{i}(u)-\eta\right|+\left(1-e^{-\pi^{2} i^{2}\left(t-t_{l}\right)}\right)\left|h_{l+1}\right| \\
\leq & \left|\int_{s}^{t} e^{-\pi^{2} i^{2}(s-u)} e_{i} \mathrm{~d} Z_{i}(u)\right|+\left|\left\langle\mathcal{Z}_{S}\left(t_{l}\right), e_{i}\right\rangle-\gamma\right| \\
& +\left|\int_{t_{l}}^{s} e^{-\pi^{2} i^{2}\left(t_{l}-u\right)} e_{i} \mathrm{~d} Z_{i}(u)-\eta\right|+\left(1-e^{-\pi^{2} i^{2}\left(t-t_{l}\right)}\right)\left|h_{l+1}\right| \\
\leq & \tilde{\varepsilon} / 8+\tilde{\varepsilon} / 8+\tilde{\varepsilon} / 8=\tilde{\varepsilon} / 2,
\end{aligned}
$$

by the fact that $\omega \in \Omega_{l}^{3}, \omega \in \Omega_{l}^{2}, \omega \in \Omega_{l}^{1}$ and by Eq. 3.12, respectively. Hence $\left|\left\langle\mathcal{Z}_{S}\left(t_{l_{1}}\right), e_{i}\right\rangle-h_{l+1}\right|<\tilde{\varepsilon}$ and

$$
\mathbf{I I} \leq \tilde{\varepsilon}\left(t_{l+1}-s\right) .
$$

Therefore,

$$
\int_{0}^{t_{l+1}}\left\langle\mathcal{Z}_{S}(t)(\omega)-f, e_{i}\right\rangle^{2} \mathrm{~d} t \leq \tilde{\varepsilon} t_{l}+\tilde{\varepsilon}\left(s-t_{l}\right)+\tilde{\varepsilon}\left(t_{l+1}-s\right)=\tilde{\varepsilon} t_{l+1} .
$$

This completes the proof.

We shall show that under some condition on the Lispchitz constant $L_{g}$ of the function $g$ the semigroup $\left(P_{t}\right)_{t \geq 0}$ satisfies the e-property. It will be also shown that this semigroup is weakly topologically irreducible.

Proposition 4 If the Lipschitz constant $L_{g} \leq \pi^{2}$, then the semigroup $\left(P_{t}\right)_{t \geq 0}$ given by Eq. 3.9 has the e-property and is weakly topologically irreducible. Consequently it admits at most one invariant measure. 
Proof If the Lipschitz constant $L_{g} \leq \pi^{2}$, then the mapping $G=\Delta-g$ is weakly dissipative. Hence $\left(P_{t}\right)_{t \geq 0}$ has the e-property.

To finish the proof we must verify irreducibility. Set $\mathcal{Z}_{S}(t)=\int_{0}^{t} S(t-s) \mathrm{d} \mathcal{Z}(s)$ for $t \geq 0$. Observe that $u_{\xi}(t)=v_{\xi}(t)+\mathcal{Z}_{S}(t)$, where $v_{\xi}$ is a continuous $\mathcal{F}_{t}$-adapted solution to the equation

$$
v_{\xi}(t)=S(t) \xi+\int_{0}^{t} S(t-s) g\left(v_{\xi}(s)+\mathcal{Z}_{S}(s)\right) \mathrm{d} s \quad \text { for } t \geq 0, \quad \mathbb{P} \text {-a.s. }
$$

If we show that for any $T>0$ and $\xi \in \mathbb{L}^{2}$ we have $\operatorname{supp} \mathcal{L}\left(u_{\xi}(T)\right)=\mathbb{L}^{2}$, we will be done. (Here $\mathcal{L}\left(u_{\xi}(T)\right)$ denotes the distribution of the random vector $u_{\xi}(T)$.) To do this fix $\xi \in \mathbb{L}^{2}$ and $T>0$. Let $v \in \mathbb{L}^{2}$. Fix $\varepsilon>0$. From Proposition 2.11 in [7] it follows that there exists $f \in \mathbb{L}^{2}\left([0, T]: \mathbb{L}^{2}\right)$ such that $\left\|v_{\xi}^{f}(T)\right\|<\varepsilon / 2$, where $v_{\xi}^{f}$ is the unique solution to the equation

$$
v_{\xi}^{f}(t)=S(t) \xi+\int_{0}^{t} S(t-s) g\left(v_{\xi}^{f}(s)+f(s)\right) \mathrm{d} s \quad \text { for } t \in[0, T] .
$$

Observe that since $g$ is Lipschitzean, the function $v_{\xi}(T): \mathbb{L}^{2}\left([0, T]: \mathbb{L}^{2}\right) \rightarrow \mathbb{L}^{2}$ is continuous. Therefore there exists $r>0$ such that if $\int_{0}^{T}\|f(t)-g(t)\|^{2} \mathrm{~d} t<r$, then $\left\|v_{\xi}^{g}(T)\right\|<\varepsilon / 2$. Further

$$
\mathbb{P}\left(\int_{0}^{T}\left\|f(t)-\mathcal{Z}_{S}(t)\right\|^{2} \mathrm{~d} t<r \text { and }\left\|\mathcal{Z}_{S}(T)-v\right\|<\varepsilon / 2\right)>0,
$$

by Lemma 3. Application of Proposition 3 completes the proof.

Open Access This article is distributed under the terms of the Creative Commons Attribution Noncommercial License which permits any noncommercial use, distribution, and reproduction in any medium, provided the original author(s) and source are credited.

\section{References}

1. Da Prato, G., Zabczyk, J.: Ergodicity for Infinite Dimensional Systems. Cambridge University Press, Cambridge (1996)

2. Doob, J.L.: Asymptotic property of Markoff transition probability. Trans. Am. Math. Soc. 64, 393-421 (1948)

3. Hairer, M., Mattingly, J.: Ergodicity of the 2D Navier-Stokes equations with degenerate stochastic forcing. Ann. Math. 164(3), 993-1032 (2006)

4. Khas'minski, R.Z.: Ergodic properties of recurrent diffusion processes and stabilization of the solutions to the Cauchy problem for parabolic equations. Theory Probab. Appl. 5, 179-196 (1960)

5. Komorowski, T., Peszat, S., Szarek, T.: On ergodicity of some Markov processes. Ann. Probab. 38(4), 1401-1443 (2010)

6. Lasota, A., Szarek, T.: Lower bound technique in the theory of a stochastic differential equation. J. Differ. Equ. 231, 513-533 (2006)

7. Maslowski, B.: Strong Feller property for semilinear stochastic evolution equations and applications. In: Stochastic Systems and Optimization. Lecture Notes in Control Inform. Sci., vol. 136, pp. 210-224. Springer, Berlin (1989)

8. Maslowski, B., Seidler, J.: Probabilistic approach to the strong Feller property. Probab. Theory Relat. Fields 118, 187-210 (2000)

9. Peszat, S., Zabczyk, J.: Strong Feller property and irreducibility for diffusions on Hilbert spaces. Ann. Probab. 23, 157-172 (1995) 
10. Peszat, S., Zabczyk, J.: Stochastic heat and wave equations driven by an impulsive noise. In: Da Prato, G., Tubaro, L. (eds.) Stochastic Partial Differential Equations and Applications-VII. A Series of Lecture Notes in Pure and Applied Mathematics, vol. 245, pp. 229-242 (2005)

11. Peszat, S., Zabczyk, J.: Stochastic Partial Differential Equations with Lévy Noise. An Evolution Equation Approach. Cambridge University Press, Cambridge (2007)

12. Priola, E., Zabczyk, J.: Structural properties of semilinear SPDEs driven by cylindrical stable processes. Probab. Theory Relat. Fields 149(1-2), 97-137 (2011)

13. Szarek, T.: The uniqueness of invariant measures for Markov operators. Stud. Math. 189(3), 225233 (2008)

14. Szarek, T., Ślęczka, M., Urbański, M.: On stability of velocity vectors for some passive tracer models. Bull. Lond. Math. Soc. 42, 923-936 (2010) 